\title{
Regular and complex singularities of the generalised thin film equation in two dimensions
}

\author{
M. Dallaston ${ }^{1}$, M. A. Fontelos ${ }^{2}$, M. A. Herrada ${ }^{3}$, J. M. \\ Lopez-Herrera $^{3}$, and J. Eggers ${ }^{4}$ \\ ${ }^{1}$ School of Mathematical Sciences, Queensland University of Technology, Brisbane, QLD, \\ 4001, Australia \\ ${ }^{2}$ Instituto de Ciencias Matemáticas, (ICMAT, CSIC-UAM-UCM-UC3M), C/ Serrano 123, \\ 28006 Madrid, Spain \\ ${ }^{3}$ E.S.I., Universidad de Sevilla, Camino de los Descubrimientos s/n 41092, Spain \\ ${ }^{4}$ School of Mathematics, University of Bristol, Fry Building, Woodland Road, Bristol BS8 \\ 1UG, United Kingdom
}

(Received xx; revised xx; accepted xx)

We use a generalised version of the equation of motion for a thin film of liquid on a solid, horizontal substrate as a model system to study the formation of singularities in space dimensions greater than one. Varying both the exponent controlling long-ranged forces, as well as the exponent of the non-linear mobility we predict the structure of the pinch-off singularity as the film thickness goes to zero. The spatial stucture of pinchoff may be either "pointlike" (approaching axisymmetry), or "quasi one-dimensional", in which case a one-dimensional singularity is unfolded into two space dimensions. The scaling of the profile with time may be either strictly self-similar (the "regular" case), or discretely self-similar and perhaps chaotic (the "irregular" case). We calculate the phase boundaries between these regimes, and confirm our results by detailed comparisons with time dependent simulations of the non-linear thin film equation in two space dimensions.

\section{Introduction}

Most of the past work on singularities has been focused on singularities in one spatial dimension. For example, in drop pinch-off one is often able to describe the dynamics in a lubrication-type description, reducing the problem to a single spatial variable $z$. The idea of self-similar solutions to this problem is that as the thickness $h$ of the neck goes to zero, $h \propto t^{\prime \alpha}$ scales like a power law of the time distance $t^{\prime}$ to the singularity (Eggers 1993). Likewise, the axial size of the singularity is assumed to scale as $z \propto t^{\prime \beta}$ as $t^{\prime} \rightarrow 0$.

Even in cases where a lubrication-type reduction is not possible, and the flow remains truly three-dimensional and axisymmetric (Chen \& Steen 1997; Day et al. 1998; Cohen et al. 1999; Zhang \& Lister 1999a; Eggers et al. 2020), the free surface shape still remains a function of one variable alone. Similarly, analytical descriptions of thin film rupture, using similarity solutions (Zhang \& Lister 1999b; Witelski \& Bernoff 2000; Craster \& Matar 2009), has been confined to the one-dimensional case (i.e. pinch-off along ridges), or axisymmetric solutions. Indeed, axisymmetric solutions have been observed in numerical simulations of the thin film equation (Becker et al. 2003; Blossey 2012), even when starting from initial conditions which are not axisymmetric themselves.

Singular structures, however, appear much more generally in two- and three dimensional fields (Eggers 2018), for example in hydrodynamic turbulence (Frisch 1995), the Euler equations (Grauer \& Sideris 1991), or the non-linear Schrödinger equation (Nore 
et al. 1997). There has been little progress describing these complex, two- or threedimensional structures analytically, although there is a general expectation that they contain self-similar features as well.

There has been some progress to extend the method of similarity solutions to higher dimensions in some specific cases, for example the porous medium equation Angenent et al. (2001); Aronson (2016), wave breaking of in a kinematic wave equation (Pomeau et al. 2008), the relativistic membrane equation (Eggers et al. 2015), in the dispersionless Kadomtsev-Petviashvili equation (Grava et al. 2016), and the formation of shocks in the compressible Euler equation (Eggers et al. 2017). Another example is the "natural focusing" of light (Nye 1999), by which a variety of higher dimensional singularities can be realised. However, a general general picture of how singularities develop in higher dimensions is missing.

Another property of complex (turbulent) flows is that they exhibit not a single feature, such as the motion toward pinch-off at a point, but a superposition of many features in a fractal arrangement. A possible such mechanism has been suggested for the Euler equation (Pumir et al. 1992), in which a self-similar solution becomes unstable to provide the seed for a new self-similar solution, a process which repeats itself on smaller and smaller scales. An explicit example of such "discrete self-similarity" has first been described for the formation of a black hole, using the equations of general relativity (Choptuik 1993). Very recently the same phenomenon has been observed in the pinch-off of a fluid, whose viscosity is much smaller than the surrounding fluid (Fontelos \& Wang 2020).

An analytical framework to understand discrete self-similarity has recently been proposed in a study of the thin film equation, in which the interaction exponent of the long ranged forces was treated as variable (Dallaston et al. 2018). As the exponent is lowered, making interactions more long-ranged, the previously self-similar pinch-off solution becomes unstable, undergoing a Hopf bifurcation, at which a periodic orbit is born. Such a periodic solution in similarity space corresponds precisely to discrete selfsimilarity, where at each cycle a new structure is produced.

In the present paper, we will provide a general framework for how singularities look like in higher dimensions, starting from the ansatz

$$
h(x, y, t)=t^{\prime \alpha} H(\xi, \eta), \quad \xi=\frac{x^{\prime}}{t^{\prime \beta}}, \quad \eta=\frac{y^{\prime}}{t^{\prime \bar{\beta}}} .
$$

If we disregard the dependence on $y$, this is the usual form of a one-dimensional similarity solution describing for example drop pinch-off, but now we allow another scaling in the transversal, $y$-direction. We find that there are two types of solutions. In the first type, which we call "pointlike", the scaling is the same in both directions, and $\bar{\beta}=\beta$. A particular case are axisymmetric solutions, which have been found to describe many cases of pinching in the thin film equation (Zhang \& Lister 1999b; Witelski \& Bernoff 2000).

In the second type of solution, the solution varies much more slowly in the transversal direction, i.e. $\bar{\beta}<\beta$. We will see that in that case, the higher dimensional dynamics results from the unfolding of a one-dimensional singular solution in the sense of catastrophy theory (Arnold 1984; Nye 1999; Pomeau et al. 2008); these will be called "quasi onedimensional solutions". We will give a general criterion for the appearance of quasi one-dimensional solutions, separating them from cases where the singularity occurs in a pointlike fashion. This idea also applies when the one-dimensional solution is no longer self-similar, but discretely self-similar or even chaotic.

We illustrate our theory using a generalized thin film equation, in which both the 
interaction exponent and the exponent of mobility appear as free parameters. Owing to the attraction between the top and bottom of the film, starting from a small sinusoidal perturbation to the film thickness $h(x, y, t), h$ goes to zero in finite time, producing a singularity in which quantities like the pressure blow up. With our theory, we are able to delineate the transitions between quasi one-dimensional and pointlike singularities on the one hand, and simple self-similar solutions and irregular solutions on the other.

To compare with theory, we present detailed numerical simulations, using two different methods, which use local refinement to be able to follow the evolution of the film thickness through three orders of magnitude, within a fully two-dimensional spatial description. We detect quasi one-dimensional, pointlike, regular, and irregular behavior, and find agreement with theoretical predictions for the phase plane. Moreover, we present detailed comparisons of the film profile between theory and simulation for all the cases considered.

In the next section, we present the generalized thin film equation, and develop the similarity theory for space dimensions larger than one, including transitions between regular and irregular behavior. The latter is accomplished by computing bifurcation curves of the one-dimensional profiles, generalizing the approach of (Dallaston et al. 2018). In the third section, we present the numerical methods used, while the fourth section contains a detailed comparison between theory and simulation. We conclude with a discussion and perspectives for the future.

\section{Self-similar solutions of the thin film equation}

\subsection{The generalized thin film equation}

The most frequently used version of the thin film equation (Craster \& Matar 2009; Blossey 2012) is that for a layer of viscous liquid on a solid substrate. It describes how viscous motion is driven by pressure gradients. In the lubrication approximation, in which the pressure $p(x, t)$ is taken to be constant over the layer, the equation for the film thickness $h(x, t)$ becomes

$$
h_{t}=\frac{1}{3}\left(h^{3} p_{x}\right)_{x}=0 .
$$

Here subscripts refer to differentiation with respect to the variable. For simplicity, we restrict ourselves to one dimension, and generalize to higher dimensions later. If the fluid is allowed to slip over the solid surface, as is the case for entangled polymer solutions (Blossey 2012), the mobility $h^{3}$ changes its power to $h^{2}$.

If it were for surface tension alone, a flat film would be a state of minimum energy, and the film would relax back to it, even if perturbed. However, for very thin films the top and bottom of the film often attract one another (Bonn et al. 2009), and pinch-off can occur. This is modelled by an additional disjoining pressure, and in the case of non-retarded van der Waals forces (Blossey 2012),

$$
p=-\gamma h_{x x}-\frac{A}{6 \pi h^{3}},
$$

where $\gamma$ is the surface tension, and $A>0$ the Hamaker constant. Other exponents for the van der Waals attraction are are also possible, for example $p \propto h^{-2}$ for retarded van der Waals forces (Blossey 2012).

Without aiming to model a particular system, but noting that exponents may differ according to experimental circumstances, we now allow the two exponents to vary freely. We also choose units of $h, x$, and $t$ such that prefactors become unity, to arrive at the 
equations:

$$
h_{t}-\left(h^{n} p_{x}\right)_{x}=0, \quad p=-h_{x x}+\frac{h^{-m}}{m} .
$$

The classical case of non-retarded van der Waals forces, and a Newtonian liquid on a solid surface, corresponds to $n=m=3$. Alternatively, this "generalized lubrication equation" (Eggers \& Fontelos 2009; Dallaston et al. 2018) may be written in the compact form

$$
h_{t}+\left(h^{n} h_{x x x}+h^{n-m-1} h_{x}\right)_{x}=0 .
$$

In the case of a two-dimensional film, which is the main subject of this paper, the lubrication equation $h_{t}+\boldsymbol{\nabla} \cdot \mathbf{f}=0$ now has the flux $\mathbf{f}=-h^{n} \boldsymbol{\nabla} p$, and the pressure is $p=-\triangle h+h^{-m} / m$. Here we have once more chosen units to normalize coefficients to unity. Now the generalized lubrication equation (Bertozzi \& Pugh 1994) in two or higher dimensions becomes

$$
h_{t}+\nabla \cdot\left(h^{n} \nabla \triangle h+h^{n-m-1} \nabla h\right)=0 .
$$

Assuming that $h(x, t)$ depends on a single spatial variable, one of course recovers (2.4).

\subsection{One dimensional similarity solutions}

We begin with the simplest case of a single spatial variable, corresponding to pinch-off of a one-dimensional ridge. We consider (2.4) and look for similarity solutions of the form

$$
h(x, t)=t^{\prime \alpha} H(\xi), \quad \xi=\frac{x^{\prime}}{t^{\prime \beta}},
$$

where $t^{\prime}=t_{0}-t$ and $x^{\prime}=x-x_{0}$. Inserting this into (2.4), and balancing the powers of $t^{\prime}$ that arise from each of the three terms, we find a unique solution for the exponents in terms of the parameters $n$ and $m$ :

$$
\alpha=\frac{1}{2+2 m-n}, \quad \beta=\frac{1+m}{4+4 m-2 n} .
$$

Using this values, we obtain an equation for the similarity profile $H(\xi)$ :

$$
-\alpha H+\beta \xi H_{\xi}=-\left[H^{n} H_{\xi \xi \xi}+H^{n-m-1} H_{\xi}\right]_{\xi} .
$$

As first found for the drop pinch-off problem (Brenner et al. 1996), and in the case of (2.8) for $n=m=3,(2.8)$ has an infinite sequence of solutions, but only one of which, the "ground state", is observed in simulation or experiment. This raises the question of the stability of such solutions, which can be studied by rewriting (2.4) in self-similar form. This is achieved by the transformation (Giga \& Kohn 1985)

$$
h(x, t)=t^{\prime \alpha} H(\xi, \tau), \quad \xi=x^{\prime} / t^{\prime \beta}, \quad \tau=-\ln t^{\prime},
$$

so that the similarity equation becomes

$$
H_{\tau}=\alpha H-\beta \xi H_{\xi}-\left[H^{n} H_{\xi \xi \xi}+H^{n-m-1} H_{\xi}\right]_{\xi} .
$$

The advantage of this "dynamical system" description (Eggers \& Fontelos 2015) is that of (2.6) are now fixed points of (2.10), making stability much easier to study. To investigate the neighborhood of a similarity solution, (2.10) has to be solved subject to the far-field condition (Eggers \& Fontelos 2015; Witelski \& Bernoff 2000)

$$
H_{\tau}=\propto \alpha H-\beta \xi H_{\xi}, \quad|\xi| \rightarrow \infty,
$$

which ensures matching to a slowly-evolving far-field solution.

Linearising (2.10) around a similarity solution, which satisfies (2.8), stability can be 
assessed within the space of one-dimensional solutions. It is found (Bernoff et al. 1998; Eggers 2012) that only the ground state is stable, all other solutions have at least one eigenvalue with a positive real part, in agreement with simulations. This excludes two positive eigenvalues (Giga \& Kohn 1985; Eggers \& Fontelos 2015), which are always present owing to the invariance of the equations under space and time translations. Namely, a perturbation will produce a shift in $t_{0}$ and $x_{0}$, leading to the solution being driven away from the fixed point, unless $t_{0}$ and $x_{0}$ are adjusted to account for this shift. The corresponding eigenvalues are $\nu_{T}=1$ and $\nu_{X}=\beta$, for the temporal and spatial shift, respectively.

The stability of (2.8) was first investigated by Witelski \& Bernoff (2000) for the case $n=m=3$, ignoring eigenvalues with imaginary part. We will see below that the most dangerous eigenvalues (those with the largest real part, apart from $\nu_{T}$ and $\nu_{X}$ ), are in fact complex. Consistent with other systems, the ground state similarity solution was found to be stable, all other solutions unstable. This remains to be true if the missing complex solutions are accounted for (Dallaston et al. 2018). We begin by studying singular solutions of the one-dimensional equation (2.4) only.

\subsection{Regular and irregular motion}

In Dallaston et al. (2017), starting from the known solution for $n=m=3$, new solutions were found by numerical continuation, holding the friction exponent fixed at $n=3$, but lowering $m$ from its typical value $m=3$, making the interaction more long-ranged. The stability of this branch of solutions was studied in Dallaston et al. (2018). Here we extend the analysis of Dallaston et al. $(2017,2018)$ to the whole interval $1 \leqslant n \leqslant 3$, as represented in the phase diagram shown in Fig. 1. We focus on the stability of the ground state solution $H_{0}(\xi)$, ignoring higher order branches $H_{i}, i \geqslant 1$, which are always unstable. In the upper part of the diagram, labelled regular, symmetric, one finds symmetric (stable) ground state solutions, an example of which is shown in Fig. 2(a), for $n=3, m=2$, and marked by green star in Fig. 1 . These solutions are similar to those found originally for $n=m=3$.

As explained in more detail in Appendix A, we are interested in solutions to the linearised problem of the form $e^{\nu \tau} P(\xi)$, subject to the far-field condition (2.11). This leads to an eigenvalue problem for $\nu=\nu_{R}+\nu_{I}$, all of which have a negative real part, making the fixed point solution $H_{0}(\xi)$ stable; this excludes the trivial eigenvalues $\nu_{T}, \nu_{X}$.

Of particular interest are the eigenvalues with the two largest real parts (both of which are complex), the larger one of which has a symmetric eigenfunction, the smaller (more negative) eigenvalue corresponding to an antisymmetric eigenfunction. As $m$ is lowered from 3, the real parts increase, to produce Hopf bifurcations when $\nu_{R}=0$ at finite $\nu_{I} \neq 0$; this signals the birth of a periodic orbit (Drazin 1992), while the original fixed point solution $H_{0}(\xi)$ has become unstable. This happens first for the symmetric eigenfunction at a value $m_{s}$, and then for the antisymmetric eigenfunction, at $m_{a}$.

Extending our numerical procedure to search for periodic solutions of (2.10) (see the Appendix A for details), we can continue the periodic solution branches from the Hopf bifurfaction, where they are created. The branch originating at the symmetric Hopf bifurcation creates symmetric periodic solutions, the asymmetric Hopf bifurcation creates antisymmetric periodic solutions.

The search for Hopf bifurcations of the ground state solution has been carried out for $1 \leqslant n \leqslant 3$, which results in bifurcation curves $m_{s}(n)$ and $m_{a}(n)$, which are drawn as the blue and yellow lines in Fig. 1, respectively. Below the blue line, the symmetric fixed point solution has become unstable, and one should expect irregular behavior: periodic, or perhaps even more complicated. As seen in Fig. 1, the symmetric Hopf bifurcation 


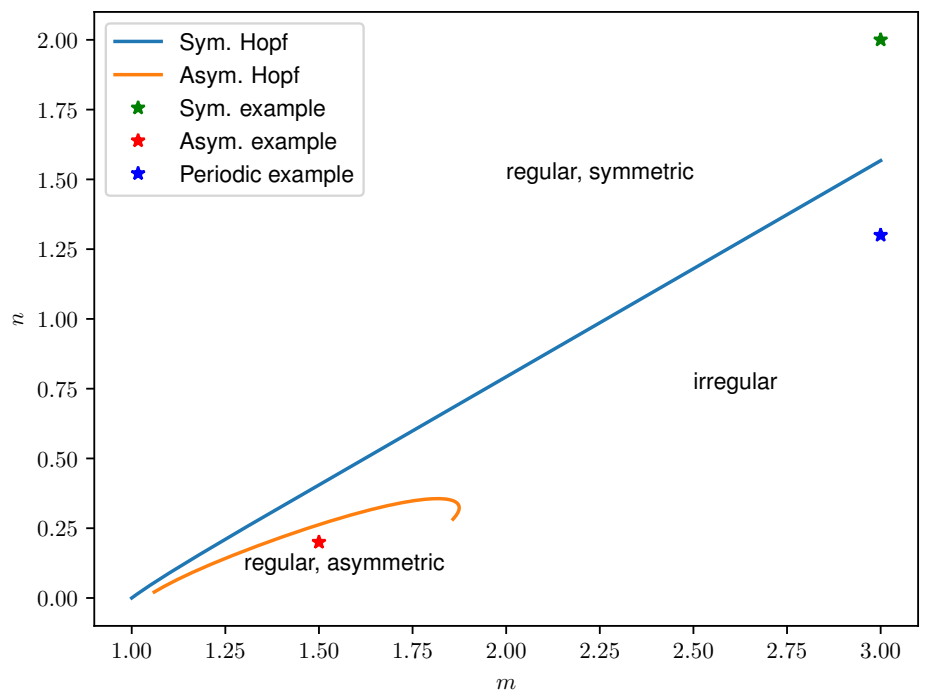

FiguRE 1. Phase diagram of one dimensional singular solutions of (2.4). Below the symmetric Hopf bifurcation (blue line), regular pinch solutions become unstable, and periodic, or even more complex dynamics appears. For small values of $n$ below $\approx 1.87$, regular, asymmetric solutions are seen below a Hopf bifurcation in the reverse direction. Examples, seen in Fig. 2 are marked by symbols. label mixed up; include antisymmetric Hopf line, put in labels

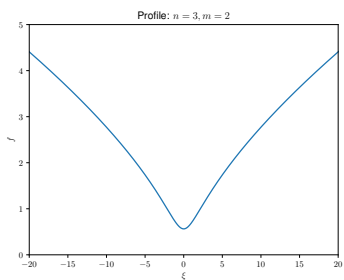

(a)

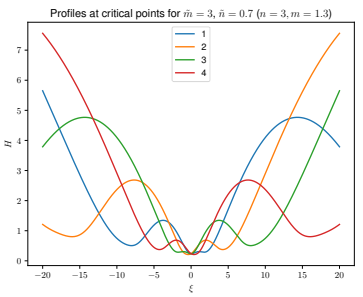

(b)

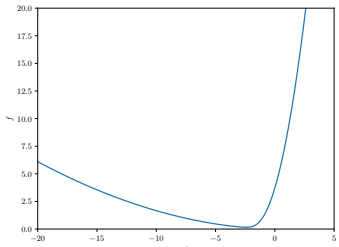

(c)

Figure 2. Three examples of similarity solutions, identified by symbols in Fig. 1. (a): a regular, symmetrix similarity solution for $n=3, m=2$ (green star) (b): a periodic, antisymmetric solution for $n=3, m=1.3$ (blue star). (c): a regular, asymmetric solution for $n=1.5, m=0.2$ (red star). The periodic solution has a period of $T=$ ??, and 5 profiles are shown, with a distance of $\Delta \tau=\ldots$ between them. need $T$; symbols and lettering needs to be much bigger; label on $\mathrm{y}$ axis is $H$. Don't need titles. put periodic $H_{\min }$ as inset in (b). make profiles (a),(c) black

line is close to linear, and well described by the equation $m=0.8(n-1)$. As a good rule of thumb, we can therefore say that

$$
m>0.8(n-1): \quad \text { regular dynamics }
$$

is the condition for a regular fixed-point solution; in the opposite case, irregular behavior will be seen.

We have not yet fully described the bifurcation structure below the asymmetric Hopf line, where both symmetric and antisymmetric solutions should be present. However, we 

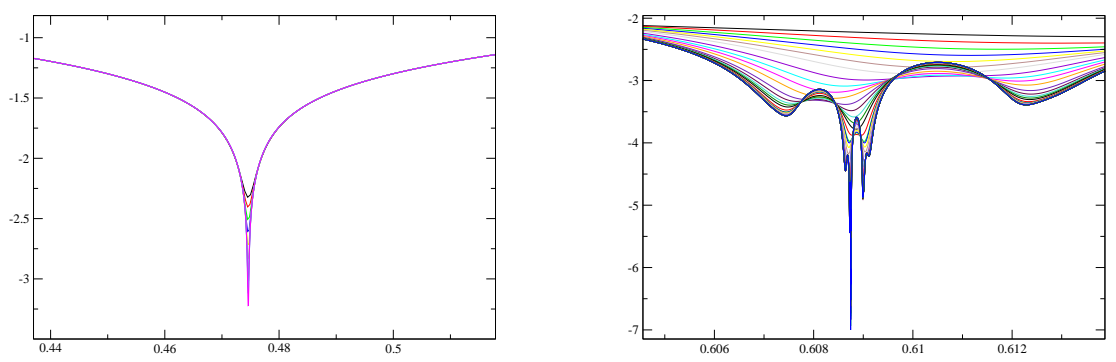

FiguRE 3. Simulations of (2.3) with $n=3, m=2$ (left, regular case), and $n=3, m=1.3$ (right, irregular case), plotting $\log _{10} h(x, t)$ as the singularity is approached. check numerics, add labels, make numbers bigger, make profiles black, and fewer of them

conclude from full numerical simulations of (2.5) that the asymmetric solution branch is an attractor. In Fig. 2(b) we show a typical example of such an antisymmetric solution, which is found by continuing the periodic solution branch from the antisymmetrix Hopf bifurcation. It corresponds to a solution $H(\xi, \tau)$ in similarity space, which is periodic in $\tau$ with period $T$, after which it returns to the same profile. Thus if profiles are recorded at discrete times $\tau_{n}=\tau_{0}+n T$, where $n$ is an integer, one observes a self-similar shrinking of the solution, hence the name "discrete self-similarity". In Fig. 2, we show a sequence of 5 profiles at equal steps of $\tau$, after one returns to the original profile.

The difference between a fixed point solution and a periodic solution is illustrated in Fig. 3, where direct numerical simulations of the one-dimensional thin film equation (2.4) are shown. On the left, we show the regular case: the same symmetric profile appears on smaller and smaller scales. On the right, we show the irregular case of a periodic orbit (discrete self-similarity). The profile undergoes a series from instabilities, continually changing the profile, but repeating the same sequence after a period $T$ in logarithmic time.

In Dallaston et al. (2018) it is shown with the example of $n=3, m=1$, that solutions can also become non-periodic. While in the discretely self-similar case the profile continually undergoes the same sequence of instabilities, in the non-periodic case new strctures are always created. While the periodic case can be seen as creating a simple fractal, where the same pattern appears on smaller and smaller scales, the non-periodic case corresponds to a multifractal. Apart from some specific examples, we have not yet delineated regions of more complicated behavior in the phase diagram.

However, as seen in Fig. 1, not all solutions below the blue bifurcation line are irregular. For $n \lesssim 1.87$, below the red bifurcation line, fixed point solutions become once more stable, but are now asymmetric, an example of which is shown in Fig. 2(c). As detailed in Appendix A, these solutions were found from a continuation procedure from the original symmetric ground state solution. Beyond the symmetric and asymmetric Hopf bifurcations, a pitchfork bifurcation leads to a pair of unstable, asymmetric solutions with complex eigenvalue; the asymmetric pair is the mirror image of one another. Continuing this branch, the real part of the eigenvalue eventually goes through zero and becomes negative in another Hopf bifurcation, but going in the opposite direction, and producing a stable, asymmetric solution. 


\subsection{Higher dimensional solutions}

We now come to the main aim of this paper, which is to understand the structure of solutions in two and higher dimensions, using the general ansatz (1.1). We first analyze the the case of $\bar{\beta}=\beta$, leading point-like solutions. Then we analyze the case $\bar{\beta}<\beta$, which results in quasi one-dimensional solutions.

\subsubsection{Pointlike solutions}

Inserting (1.1) with $\bar{\beta}=\beta$ into the generalized thin film equation (2.5), we obtain the similarity solution

$$
-\alpha H+\beta \xi H_{\xi}+\beta \eta H_{\eta}=-\nabla \cdot\left(h^{n} \nabla \triangle h+h^{n-m-1} \nabla h\right),
$$

where $\nabla=\left(\partial_{\xi}, \partial_{\eta}\right)$, and $\triangle=\partial_{\xi}^{2}+\partial_{\eta}^{2}$. In view of spatial isotropy, one expects solutions of (2.13) to be axisymmetric (Zhang \& Lister 1999b; Witelski \& Bernoff 2000). The scaling of the exponents (2.7) is the same as in the one-dimensional case. Looking for radially symmetric solutions

$$
h(x, y, t)=t^{\prime \alpha} H(\zeta), \quad \zeta=r / t^{\prime \beta},
$$

one finds in two dimensions:

$$
-\alpha H+\beta \zeta H^{\prime}+\frac{1}{\zeta}\left[\zeta H^{n}\left(\frac{\left(\zeta H^{\prime}\right)^{\prime}}{\zeta}\right)^{\prime}+H^{n-m-1} H^{\prime}\right]^{\prime}=0 .
$$

Solutions to (2.15) have been computed for $n=m=3$ (Zhang \& Lister 1999b), and have been found to be stable to radial as well as non-axisymmetric perturbations (Witelski \& Bernoff 2000). However, the stability analysis again excluded complex eigenvalues. We will see below that axisymmetric solutions are in fact stable only for a range of $n, m$ values.

It is worth noting that pointwise similarity solutions are not necessarily axisymmetric. An example are optical caustics, which are described by the eikonal equation (Nye 1999). In that case, radially symmetric solutions would correspond to perfect focusing, and would be the least generic situation. Instead, the most stable solutions, which take the fewest number of adjustable parameters to find, are the elliptic and hyperbolic umbilic. They can be found by solving the analogue of (2.15) for the eikonal equation (Eggers 2020).

\subsubsection{Quasi one-dimensional solutions}

The other possible solution of the form (1.1) is the case $\bar{\beta}<\beta$, which means that the solution is varying slowly in the transversal direction; the direction in which the solution is most singular (largest derivatives), we choose as the $x$-direction. We have investigated solutions of this type previously in the case of the eikonal equation (Eggers et al. 2015), the dispersionless Kadomtsev-Petviashvili equation (Grava et al. 2016), and shock formation in the compressible Euler equation (Eggers et al. 2017). Here we point out the generality of the approach, which is independent of the particular structure of the equation, and apply it to the generalized thin film equation.

Owing to the slow variation in the $y$ direction, we can look at the solution as a superposition of one-dimensional solutions (2.6). Since the eigenvalue $\nu_{T}$ is usually the most singular one, we consider a corresponding shift in the singularity time of the onedimensional solution. As $y$ is varied, the singular time $t_{c}(y)$ effectively varies (Pomeau et al. 2008; Grava et al. 2016). Setting the origin of the transversal variable such that the singularity occurs for $y=0$ first, and expanding, we have

$$
t_{c}=t_{0}+a y^{2}+O\left(y^{3}\right)
$$


we must have $a>0$ so that always $t_{c} \geqslant t_{0}$. For the same reason, there can be no linear term. Replacing $t^{\prime}$ in (2.6) by $t_{c}-t=t^{\prime}+a y^{2}+O\left(y^{3}\right)$ implies the scaling $y^{2} \propto t^{\prime}$, and thus $\bar{\beta}=1 / 2$. The singularity proceeds in the $x$-direction, but is "unfolded" in the transversal (y) direction, a situation well understood in the case of the eikonal equation (Nye 1999; Eggers et al. 2015). The similarity equation of the thin film problem then becomes

$$
-\alpha H+\beta \xi H_{\xi}+\frac{\eta}{2} H_{\eta}=-\left[H^{n} H_{\xi \xi \xi}+H^{n-m-1} H_{\xi}\right]_{\xi} .
$$

Note

that in order for (2.16) to be self-consistent, we must have $\beta>1 / 2$. In that case the $x$-derivatives on the right hand side of (2.5) dominate over the $y$-derivatives, and the right-hand-side only contains $\xi$-derivatives. Combining the condition $\beta>1 / 2$ for a quasi-one-dimensional singularity with the formula for $\beta$, one obtains

$$
n>1+m, \quad \text { quasi-one-dimensional }
$$

as a necessary condition for a quasi one-dimensional singularity. The same stability boundary is obtained by considering a small $y$-dependent perturbation to a onedimensional solution (Witelski \& Bernoff 2000), realising that after a shift in the $y$-direction, it is enough to consider quadratic perturbations to leading order.

With the above insight, we use (2.16) directly to find the most general unfolding of a one-dimensional solution $H^{(1)}(\xi)$ of $(2.8)$, it is easy to check that

$$
H(\xi, \eta)=\left(1+A \eta^{2}\right)^{\alpha} H^{(1)}\left(\frac{\xi+B \eta^{2 \beta}}{\left(1+A \eta^{2}\right)^{\beta}}\right)
$$

is a solution of (2.16) for arbitrary constants $A, B$. They are adjustable parameters, which depend on initial conditions.

In fact, $(2.18)$ can be understood from noticing that since $H_{1}(\xi)$ does not contain any free parameters, the only way a dependence on $y$ can come in is through $t_{0}(y)$ and $x_{0}(y)$. Then to leading order $t_{c}-t=t^{\prime}+A y^{2}=t^{\prime}\left(1+A \eta^{2}\right)$. Further, we expand

$$
x-x_{c}=x^{\prime}-\sum_{i=1}^{\infty} b_{i} y^{i}
$$

so that

$$
\frac{x-x_{c}}{t^{\prime \beta}}=\xi-\sum_{i=1}^{\infty} b_{i} \eta^{i} t^{i / 2-\beta} .
$$

This only leads to a finite result if $2 \beta$ is an integer, so that in fact the contribution $B \eta^{2 \beta}$ in (2.18) is regular. All coefficients $b_{i}$ with $i / 2-\beta<0$ must vanish (otherwise they would blow up), and so the leading contribution is that of (2.18).

The form of the solution (2.18) is a general feature, independent of the structure of the equation. In the case of additional symmetries, it might even have a more general form. For example, considering the cusp singularity (Nye 1999) of the eikonal equation, and using the equivalent of (2.18), one finds a solution with three independent parameters. Only after invoking an additional invariance of the eikonal equation, does one recover the required four parameters.

In the time-dependent case, important for describing the irregular behavior discussed in Subsection 2.3, the equation extending (2.16) is

$$
H_{\tau}=\alpha H-\beta \xi H_{\xi}-\frac{\eta}{2} H_{\eta}-\left[H^{n}\left(H_{\xi \xi}-\frac{1}{m H^{m}}\right)_{\xi}\right]_{\xi} ;
$$




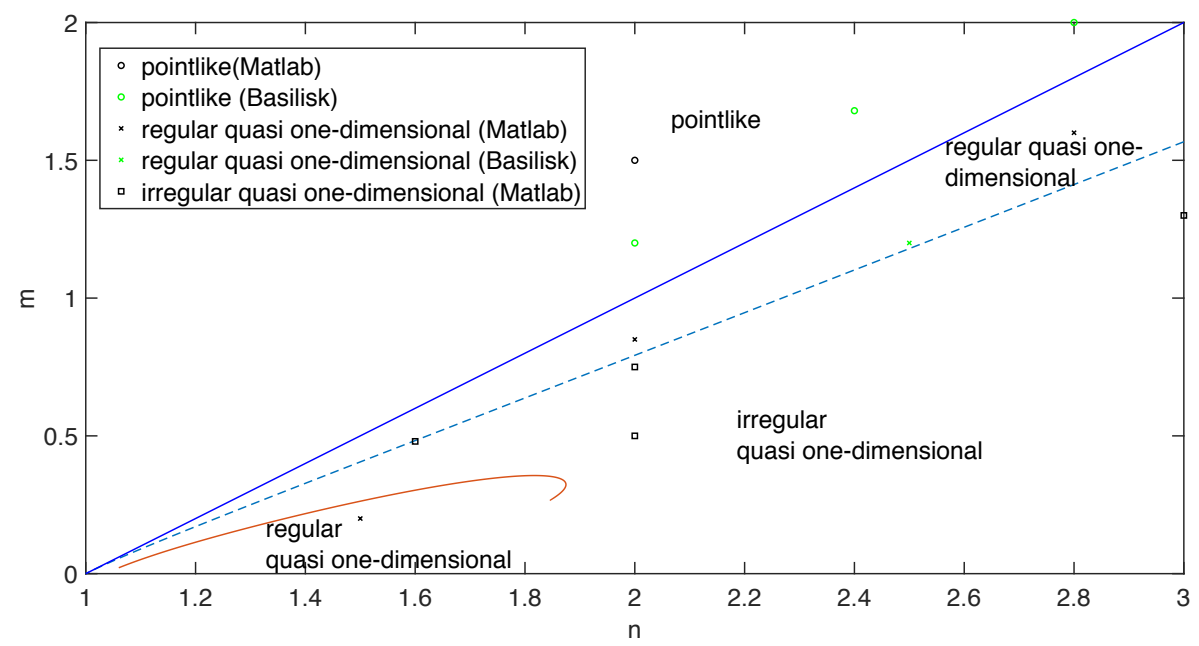

Figure 4. Phase plane of the two-dimensional singularities of (2.5). The blue solid line is the border (2.12) between regular and complex behavior, the blue dashed line is the border (2.17) between pointlike and quasi-1D behavior. For smaller values of $m$, and $n \lesssim 1.87$, there is a return to regular behavior. The symbols correspond to numerical simulations of (2.5) with initial condition (3.1).

now if $H^{(1)}(\xi, \tau)$ is a solution of $(2.10)$,

$$
H(\xi, \eta)=\left(1+A \eta^{2}\right)^{\alpha} H^{(1)}\left(\frac{\xi+B \eta^{2 \beta}}{\left(1+A \eta^{2}\right)^{\beta}}, \frac{\tau}{1+A \eta^{2}}\right)
$$

solves (2.19). Note that depending on $y$, the solution is found in different phases of the evolution. In the case of a non-periodic solution, this will be even more complicated.

\section{Numerics of the thin film equation}

In the light of the above discussion, we want to explore numerical solutions of (2.5), to see whether one can identify the types of solutions described above, and find the transitions between them. Namely, transitions between quasi 1D and pointlike solutions, and between regular and irregular solutions, as the exponents $n, m$ are varied. For simplicity we impose periodic boundary conditions on $(x, y) \in[0,1] \times[0,1]$. As initial conditions we choose

$$
h_{0}(x, y)=h_{\text {ref }}\left[1-\epsilon_{1} \cos 2 \pi(x-1 / 2)\right]\left[1-\epsilon_{2} \cos 2 \pi(y-1 / 2)\right],
$$

which helps explore transitions between one and two dimensions. If $\epsilon_{2}=0$, there is no $y$-dependence, and solutions are strictly one-dimensional. If on the other hand $\epsilon_{1}=\epsilon_{2}$, there is a single minimum at the center $(x, y)=(1 / 2,1 / 2)$ of the domain, around which the profile is approximately axisymmetric. The amplitude $h_{r e f}$ was chosen such that a flat profile is linearly unstable.

In view of the demands on the spatial resolution in two dimensions, two different numerical codes have been used in this work, using different schemes for the adaptive regridding. The first one, implemented on MATLAB (C), is based on the work of Herrada \& Montanero (2016), while the second is constructed on the platform Basilisk (Popinet 2015). 


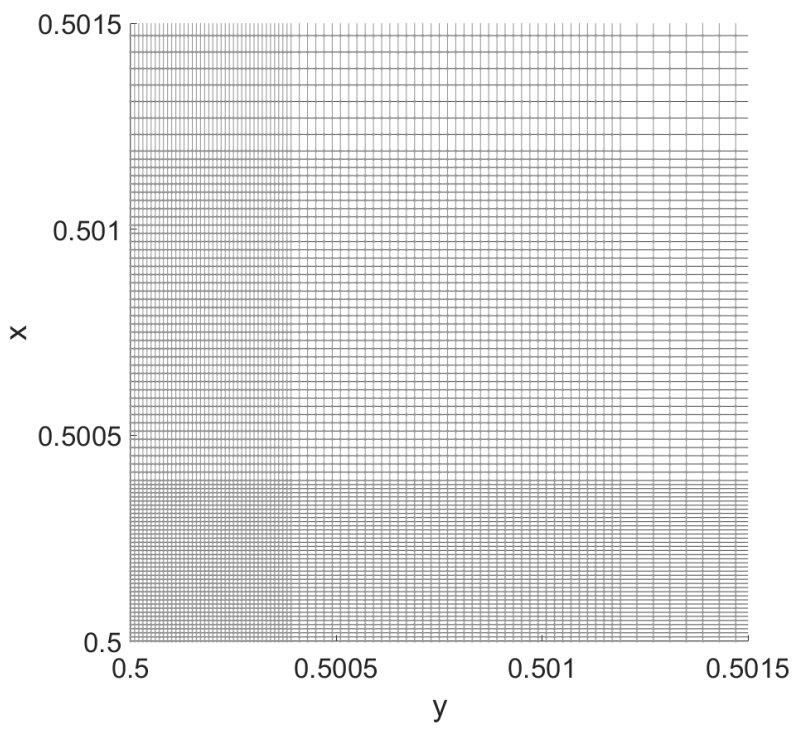

Figure 5. An example of a non uniform mesh created in the late stages of a simulations, with the minimum of $h$ at $(0.5,0.5)$.

\subsection{Fully implicit finite difference method}

In this scheme we have taken advantage of the symmetric of the problem to simulate just a quarter of the domain: $[0.5] \times[0.5]$. The two-dimensional generalized thin film equation (2.5) leads to a nonlinear equation for $h$ :

$$
\begin{aligned}
h_{t} & =h^{n}\left[h_{y y y y}+2 h_{x x y y}+h_{x x x x}+\left(h_{y y}+h_{x x}\right) / h^{m+1}-\left(h_{y}^{2}+h_{x}^{2}\right)(m+1) / h^{m+2}\right. \\
& \left.+h_{y} h^{n-1} n\left(h_{y y y}+h_{x x y}+h_{y} / h^{m+1}\right)+h_{x} h^{n-1} n\left(h_{y y x}+h_{x x x}+h_{x} / h^{m+1}\right)\right] .
\end{aligned}
$$

The time derivative is discretized using second order backward differences while second order central differences were employed to discretize the spatial derivatives. The method is fully implicit; to solve the nonlinear system resulting from the discretization, a NewtonRaphson technique was used, where the required Jacobian matrix is obtained by combining analytical functions and collocation matrices (see Herrada \& Montanero (2016) for further details). This allows to take advantage of the sparsity of the resulting matrix, to reduce the computational time to invert it.

A variable time step $d t$, based on the change in the minimum of $h$, was used for the time integration. The minima $h_{m i n}$ and $h_{m i n_{1}}$ at the current and previous time steps, respectively, as well as the last time step, $d t_{1}$, are used to set

$$
d t_{t r y}=\left(\alpha h_{m i n}-h_{m i n 1}\right) /\left(h_{m i n}-h_{m i n 1}\right) d t_{1}-d t_{1} .
$$

Here $\alpha$ is a parameter to control the variation of the time step. The step $d_{t r y}$ is used when the solution approaches the singularity, while a fixed time step $d t_{\text {fixed }}$ is used at the beginning of the simulation. To that end, the time step was set as $d t=\min \left(d t_{\text {try }}, d t_{\text {fixed }}\right)$. Most of the simulations where carried out with $\alpha=0.9$ and $d t_{\text {fixed }}=0.001$.

All simulations where started with an uniform grid with a grid size $\Delta x=\Delta y=$ 0.002. Once the solution approaches the singulary, the mesh is automatically adapted to concentrait points around the coordinates, $\left(x_{\min }, y_{\min }\right)$, where $h$ reaches its minimum value. Near this point a uniform grid with spacing $\Delta x=\Delta y=\Delta_{\min }$ is used. Starting at this point and after each $N$ steps, the grid size was doubled in each direction. The 
simulations were carried out with $\Delta_{\min }=510^{-5}$ and $N=40$. Fig. 5 shows an example of the mesh generated using this procedure.

\subsection{Basilisk library}

Alternatively, the nonlinear system of equations is solved with the Newton-Raphson scheme implemented in the free C-language library Basilisk (Popinet 2015). This allows us to make use of the sophisticated adaptive grid refinement schemes available for Basilisk, and to control the discretization error directly. Basilisk provides tools for the numerical solution of PDE's, discretized spatially with adaptive Cartesian meshes. Here, starting from a trial solution $\left(p^{*}, h^{*}\right)$, we iteratively solve the linear system

$$
\begin{aligned}
p+h_{x x}+h_{y y} & =0, \\
\left(\alpha p_{x}\right)_{x}+\left(\alpha p_{y}\right)_{y}+\left(\beta h_{x}\right)_{x}+\left(\beta h_{y}\right)_{y}+\left(\gamma_{x} h\right)_{x}+\left(\gamma_{y} h\right)_{y} & =h_{t}+\left(\gamma_{x} h^{*}\right)_{x}+\left(\gamma_{y} h^{*}\right)_{y},
\end{aligned}
$$

with

$$
\alpha=\left(h^{*}\right)^{n}, \beta=-\left(h^{*}\right)^{n-m-1} \text { and } \gamma_{x, y}=n\left(h^{*}\right)^{n-1} p_{x, y}^{*}-(n-m-1)\left(h^{*}\right)^{n-m-2} h_{x, y}^{*}
$$

up to the convergence, using a multigrid method. Here $p=p^{*}+\delta p$ and $h=h^{*}+\delta h$, with $(p, h)$ the converged solution, and $(\delta p, \delta h)$ a small correction. Convergence is checked by monitoring $\delta h$ a posteriori.

Spatial derivatives are discretized using second order differences, while the time derivative has been discretized using first or second order backward differences; no significant difference was found between both time time discretization methods. The timestep has been set according to two different strategies; it is either set proportional to the minimum height, or by estimating the time truncation error, calculated by comparing the solution obtained with second and first order time derivatives. The equations are very stiff, and prone to numerical divergence if the timestep is not small enough. Therefore, both strategies require some fine tuning of parameters, in order to approach the singularities as closely as possible. The spatial discretization is adapted automatically by monitoring the inverse of the height field, $h_{i}=1 / h$. In the vicinity of the singularities grid as small as $\Delta x=\Delta y=3 \cdot 10^{-5}$ is used while the grid size grows up $\Delta x=\Delta y=7.8 \cdot 10^{-3}$ far away of the singularities.

\section{Comparison of theory and simulation}

We are now able to compare our theoretical predictions of Fig. 4 to numerical simulations. Owing to the adaptive capabilities of both of our numerical codes, we are able to follow the dynamics through almost five orders of magnitude in scale check. This allows us to identify the self-similar structure, and classify the type of singularity according to our theoretical description. Both of our numerical codes provided consistent results.

The symbols in the phase plane of Fig. 4 correspond to simulations at set parameter pairs $(n, m)$, and are seen to agree nicely with theoretical predictions. The classification of the dynamics is based on the identification of the theoretically predicted structure. This is either the pointlike solution (2.14), or the quasi one-dimensional solution (2.18) in the regular, fixed-point case, or (2.20) in the irregular, time-dependent case. We will now go over a few representative cases, for which we present a detailed comparison.

The case of Fig. 6 is in the pointlike (axisymmetric) regime, shown as the black circle at $n=2, m=1.5$ in the phase diagram. As seen on the left of Fig. 6, although the initial condition is not axisymmetric, the solution converges to a point, with radial symmetry. To demonstrate this more clearly, we demonstrate collapse of the profiles in the $x$ and $y$ 

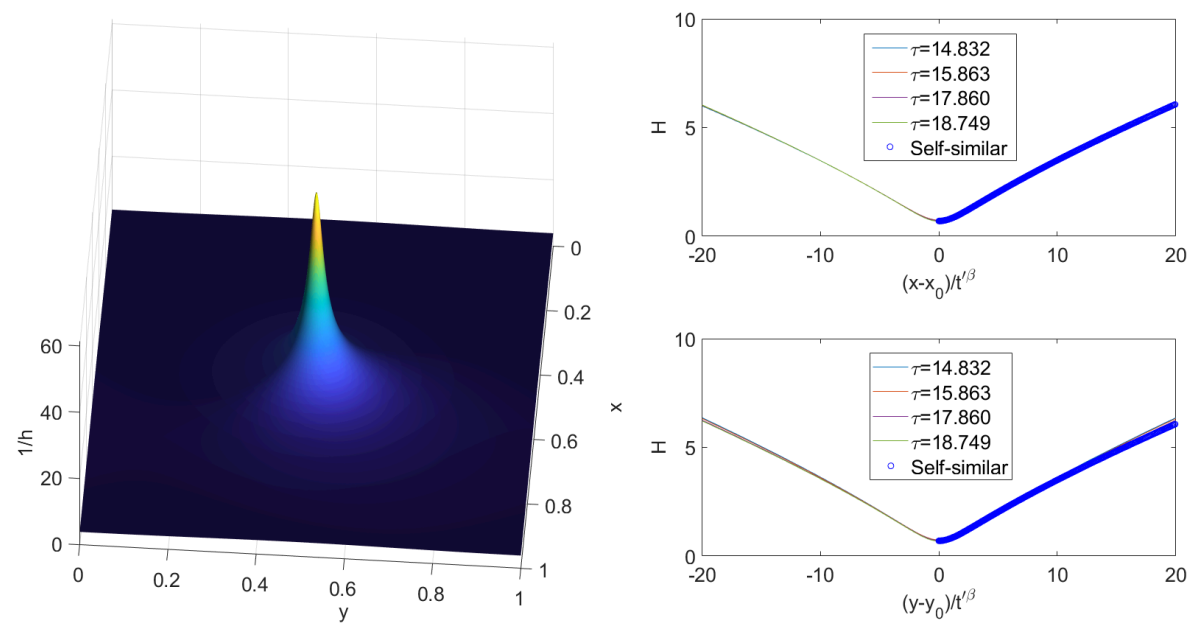

Figure 6. Simulation of (2.5) with $n=2, m=1.5$, and initial condition (3.1), using $\epsilon_{1}=0.05, \epsilon_{2}=0.03$, and $h_{\text {ref }}=0.2$. On the left, a perspective plot of $1 / h$ demonstrates the pointlike character. On the right, cuts in the $x$ and $y$ directions are shown at the top and bottom, respectively, for the values of $\tau=-\ln t^{\prime}$ shown. Profiles are collapsed according to (2.14), and agree with a solution of (2.15) (symbols), demonstrating axisymmetry.
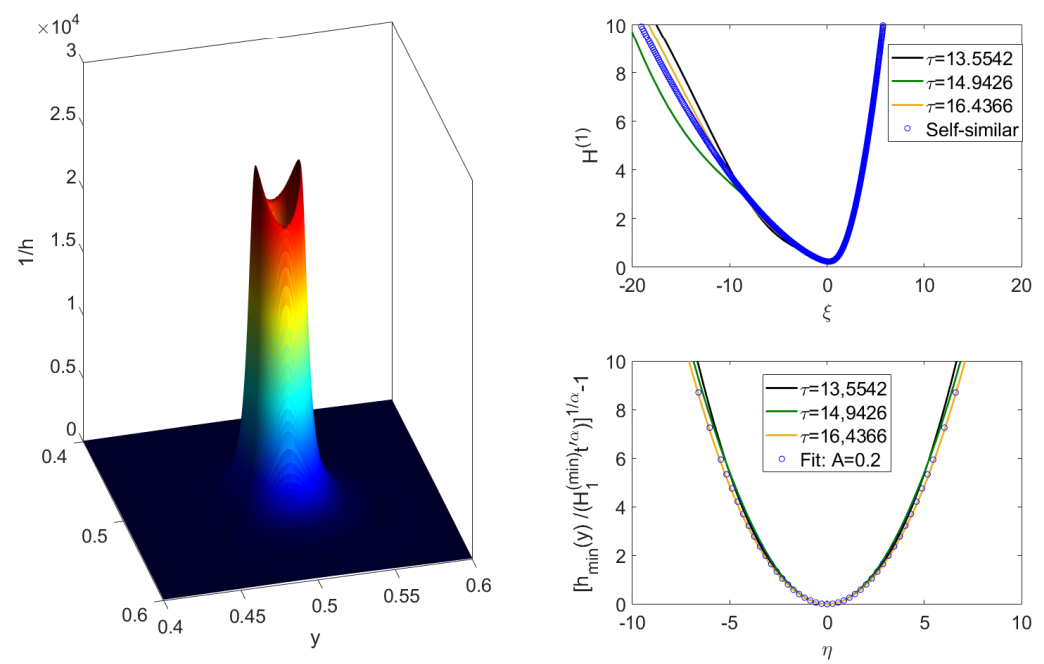

FiguRE 7. Simulation of (2.5) with $n=1.5, m=0.2$, and initial condition (3.1), using $\epsilon_{1}=0.05, \epsilon_{2}=0.03$, and $h_{r e f}=0.04$. A quasi one-dimensional, regular singularity is observed. On the left, a plot of $1 / h$ with two qusi-1D peaks; on the top right, a collapse of the profiles using (1.1), compared to (2.18). Lower left, transversal collapse using (4.1).

directions on the right. Cuts in both directions are rescaled according to (2.14). In both cases one observes very good collapse, and very good agreement with the solution to the axisymmetric similarity equation (2.15). We find the same point-like behavior for all the cases above the blue dashed line.

Next we look at a quasi one-dimensional case, shown in Fig. 7. Choosing $n=2, m=0.5$ 


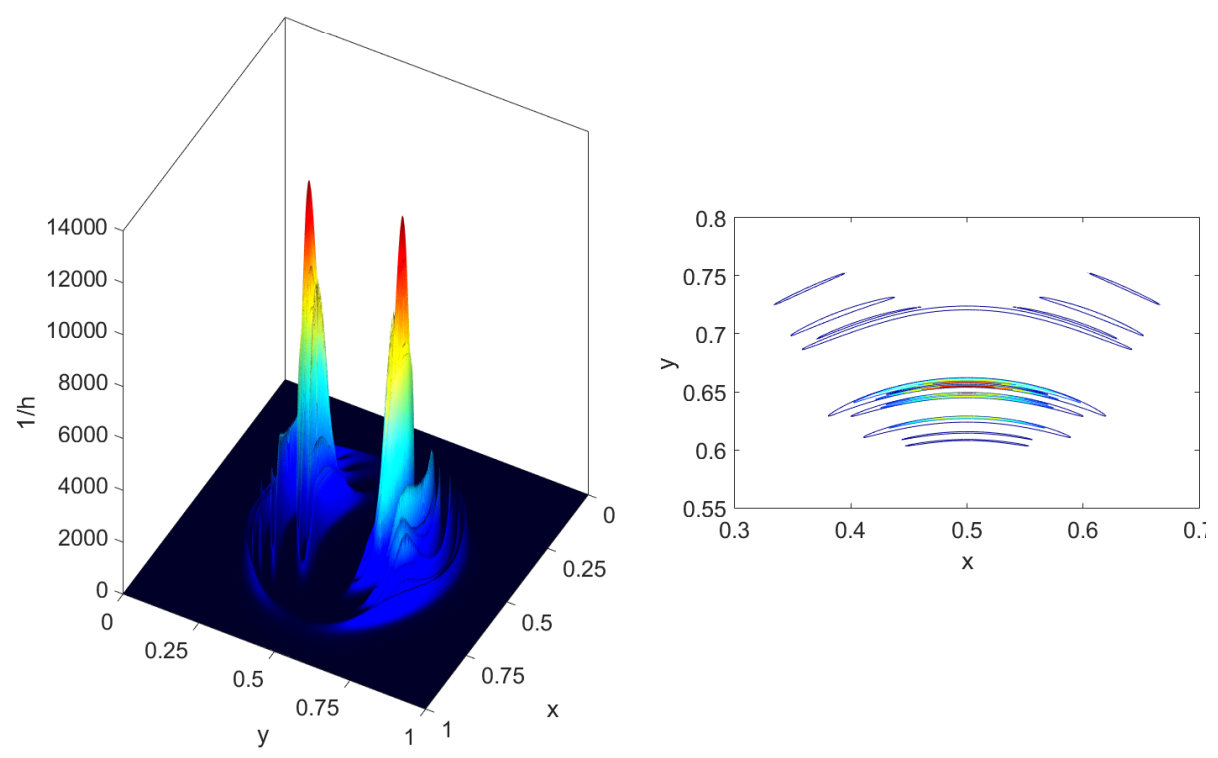

Figure 8. Simulation of (2.5) with $n=3, m=1.3$, and initial condition (3.1), using $\epsilon_{1}=0.05, \epsilon_{2}=0.03, h_{r e f}=0.1$. A quasi one-dimensional, irregular singularity results. On the left a perspective plot of $1 / h$. Along the one-dimensional front, one onserves a sequence of instabilities. On the right, a contour plot of one of the peaks of $1 / h$ shows the irregularity of the profile.

(black cross in the phase diagram), we begin with the simpler case of regular fixed point dynamics, found underneath the orange curve, which marks the reverse Hopf bifurcation; the one-dimensional profiles are highly asymmetric, as shown in Fig. 2(c). On the left of Fig. 7 , we once more plot $1 / h$ over the $(x, y)$-plane, and indicate contours by color. Owing to the periodicity of the initial condition, we now see two equal peaks, which are extremely anisotropic. Looking at the front and back of the peak, one appreciates the asymmetry of the profile in the $x$-direction. In the $y$-direction, on the other hand, the solution is unfolded: the peak is highest along the centerline $y=0.5$, and with increasing $|y-0.5|$, one sees the singularity at earlier stages of its evolution.

On the right the structure of the singularity, as described by (2.18), is analyzed more quantitatively. In the generic case of $2 \beta$ not being an integer $(\beta=3 / 4$ in the example), we have $B=0$, and it remains to calculate $A$. To that end, we calculate the minimum $H_{\text {min }}^{(1)}$ of the one-dimensional similarity profile by solving the one-dimensional similarity equation (2.8). This is the cyan curve on the top right of Fig. 7. Now we calculate the minimum of $h$ for different values of $y$. From (2.18) it follows that

$$
\left(\frac{h_{\min }(y)}{H_{1}^{(\min )} t^{\prime \alpha}}\right)^{1 / \alpha}-1=A \eta^{2},
$$

and so $A$ is found from plotting the left hand side as a function of $\eta$. This is shown on the lower right of Fig. 7, showing a collapse for different values of $\tau$; from a fit to the quadratic profiles we find $A=0.3$. Now we can test for the collapse of the whole profile using (2.18), as shown on the upper half on the right of Fig. 7. Once again, there is a good collapse and agreement with the self-similar solution.

Finally, we study a irregular, complex case, for which the dynamics is always quasi 

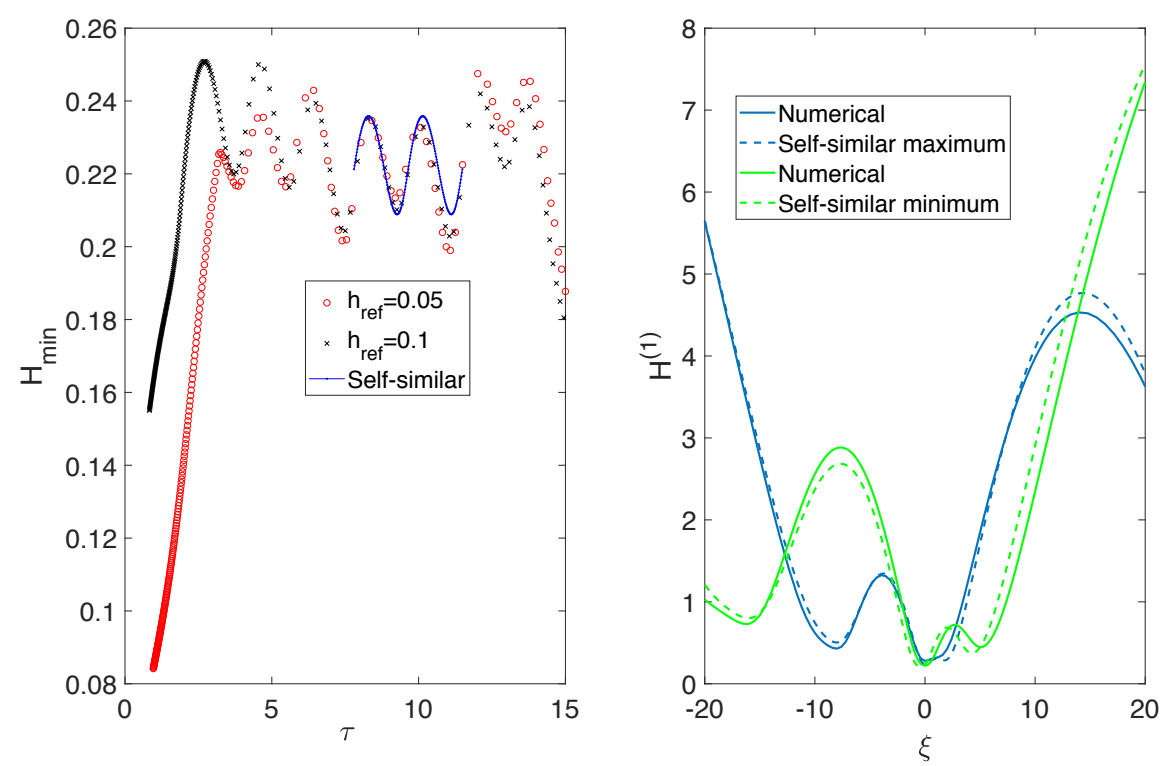

Figure 9. A quantitative test of (2.20), valid for the dynamical case. Parameters are the same as in Fig. 8. The one-dimensional, periodic profile is shown in Fig. 2(c). On the left, we plot the minimum of $t^{\prime-\alpha} h$, along the centerline $y=0$ of the profile, compared with the theoretical prediction, with $T=$ ??. On the right, the profile along the centerline is compared to the theoretical prediction. can we that as a function of $y$, similar to Fig. 7 ? what is $h_{r e f}$ ?, should be $-\ln t^{\prime}$ on $\mathrm{x}$-axis. Show two periods. Get rid of titles. is it $\epsilon_{2}=0.3$. make symbols bigger. make colors easier to distinguish

one-dimensional, since the blue Hopf bifurcation line lies underneath the dashed line, below which we observe quasi one-dimensional behavior. An example of a simulation of (2.5) is shown in Fig. 8, where $n=3$ and $m=1.2$, so according to (2.12), we are in in irregular regime. On the left hand side, we show a perspective plot of $1 / h$, and emphasize contour lines using a color code. While the peaks are smooth in the regular case, seen on the left of Fig. 7, they are now broken up into many smaller peaks, producing a spatially "spotty" behavior. In the $x$-direction, one observes the result of multiple instabilities, as seen on the right of Fig. 3 for the one-dimensional case. In addition, as $y$ is detuned from 0.5 , this irregular behavior is seen in different phases of its evolution, producing the hierarchy of peaks seen in Fig. 8. To emphasize the resulting spatial "spottiness", on the right of Fig. 8 we also represent $1 / h$ as a color plot in the plane.

In Fig. 9, we test the expected form (2.20) more quantiatively. The one-dimensional periodic orbit, calculated from (2.10), was shown in Fig. 2(c). In our two-dimensional simulation, $h$ has been rescaled according to (1.1), in order to obtain the self-similar profile. On the left, we show the overall minimum of the profile, which should vary periodically, with period $T=$ ?. The theoretical prediction is shown as the blue curve on the left of Fig. 9, which agrees very well with the numerical simulation for two periods, until we run out of numerical resolution.

To test the shape of the entire profile in its time evolution, we consider a cut through the profile at $y=0$, rescaled according to (1.1). For simplicity, we record the shape of the profile when $H_{\min }$ is maximal and minimal, corresponding to the local maximum and minimum during the oscillation seen on the left of Fig. 9. The profile from a full 


$$
n=3, m=1,2, h_{\text {ref }}=0,1
$$

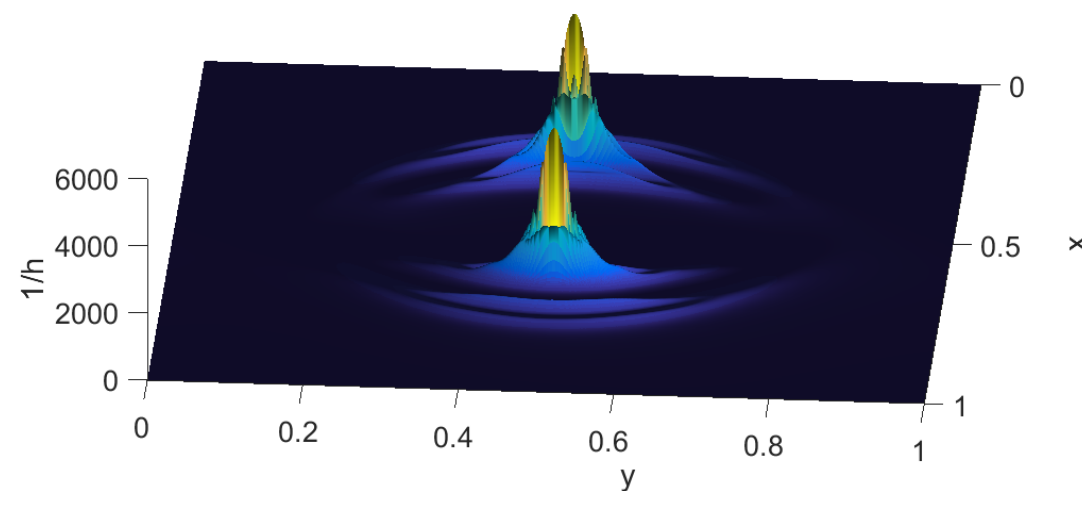

FIGURE 10. Simulation of (2.5) with $n=3, m=1$, and initial condition (3.1), using $\epsilon_{1}=0.05, \epsilon_{2}=0.03$. We show a color plot of $1 / h$. to do.

numerical simulation of (2.5) thus obtained (solid lines), is compared to the theoretical profiles on the right; very good agreement is found.

\section{Discussion}

In this paper we have pointed out generic structures of singularities in more than one dimension. The flexibility of the generalized thin film equation is useful to test many different scenarios using just one equation, but the features of higher dimensional singularities we observed here should carry over to many other equations as well. As examples of other systems we have mentioned the porous medium equation, the eikonal equation, and the compressible Euler equation.

When the interaction exponent $m$ is large, interactions are very localized, and pinching occurs in a localized or "pointlike" fashion, as had been observed previously. More complicated dynamics occurs if the interaction becomes more long-ranged, as $m$ becomes smaller. First, the higher dimensional dynamics becomes an unfolding of one-dimensional dynamics, making it a superposition showing the singularity in all its phases. Second, the underlying one-dimensional singularity may become irregular, producing new structures as smaller and smaller scales are reached.

We hope that a combination of both effects may contribute to the understanding of turbulent fields, which are characterized by complexity both in scale and in space. As an illustration, in Fig. 10 we show a snapshot of $1 / h$ for a simulation of (2.5) with $n=3, m=1$. In that case, the one-dimensional dynamics is no longer merely periodic, but new structures keep being generated as one evolves toward scales. Owing to the mechanism of unfolding, these new structures are translated into space, producing a spatially complex picture. In Fig. 10 we show a larger spatial region, incompassing several periods of the initial perturbation. Since the evolution depends sentively on the initial condition, slight changes in the initial condition translate into non-periodic behavior on the small scale. 


\section{Appendix A. Similarity solutions and their stability}

Here we apply the methods devloped in Dallaston et al. $(2017,2018)$ to a range of $n$-values. Going beyond the original papers, we use a new method to compute periodic solutions directly, and find a novel branch of asymmetric solutions. The computations are performed in the numerical continuation software AUTO07-P.

We implement our search for solutions using two different approaches: In the first method, used in Dallaston et al. $(2018,2017)$, we solve the linearized problem. We code explicitly the fourth order ODE (2.8) for the similarity profiles, as well as the ODE for the eigenfunctions, as a system of first order ODEs. This method is highly accurate and efficient, as AUTO's accurate collocation method is used to compute the derivatives in $\xi$.

In the second method, we solve the nonlinear dynamical equation (2.10) in scaled form. It is discretised in $\xi$ using finite differences, and coded into AUTO. This method is advantageous in that we now compute stability in a natural way from the Jacobian of the system, and can explicitly compute branches of periodic (i.e. discrete self-similar) solutions. However, this method is not as efficient, as AUTO is not designed to exploit the sparse/banded Jacobian structure that arises in discretised PDEs, and we have not been able to explore the complete structure of periodic solution branches.

\section{A.1. Computation of solution branches by numerical continuation}

In this appendix, we introduce the notation $\bar{n}=n-m-1$ for brevity. Rearranging (2.8) for the fourth derivative, we obtain

$$
H^{(\mathrm{iv})}=H^{-n}\left(\alpha H-\beta \xi H^{\prime}\right)-n H^{-1} H^{\prime} H^{\prime \prime \prime}-H^{\bar{n}-n} H^{\prime \prime}-\bar{n} H^{\bar{n}-n-1} H^{\prime 2}+\delta \xi \mathrm{e}^{-\xi^{2}},
$$

where $\delta$ is the strength of an added symmetry breaking term, which later will be taken to zero.

To find the first two branches $H_{0}$ and $H_{1}$ of symmetric solutions, we put $\delta=0$, and use the first method, starting from the known van der Waals solution at $n=3, \bar{n}=$ -1 (Zhang \& Lister 1999a; Witelski \& Bernoff 1999)). These branches (shown in blue in Fig. 11(a),(c)) are continued by varying $\bar{n}$, until they merge at a fold singularity (Dallaston et al. 2018, 2017), marked by a circle. These branches are then continued to all $1 \leqslant n \leqslant 3$. The cases $n=3$ and $n=1.5$ are shown in Fig. 11(a) and (c), respectively. By varying $\bar{n}$, the fold singularity traces out a line in the $(n, \bar{n})$ plane, shown as the purple line in Fig. 12.

The symmetry breaking term with $\delta>0$ is introduced to find asymmetric solutions. When $\bar{n}$ is decreased, the $H_{0}$ solution branch passes through a pitchfork bifurcation, at which an asymmetric branch originates, which consists of pairs of solutions, one a mirror image of the other. Once on the asymmetric branch, $\delta$ can be taken to zero again, and the solution branch traced out by varying $\tilde{n}$, shown as the orange branch in Fig. 11(a),(c)). This can once more be continued to all $1 \leqslant n \leqslant 3$, exhibiting the same structure; the pitchfork bifurcation is shown as the green line in Fig. 12.

\section{A.2. Stability and Hopf Bifurcations}

Using the first, linear method, the stability of similarity solutions is determined by solving the coupled system of equations for the eigenfunctions, as well as for the similarity profiles. The real and imaginary parts of the eigenvalue are used as continuation parameters. This approach was performed in Dallaston et al. (2018), where the Hopf bifurcations that leads to discrete self-similarity was first computed.

There are two Hopf bifurcations, as seen in Fig. 11(a),(c)): as $\bar{n}$ is increased, first one 


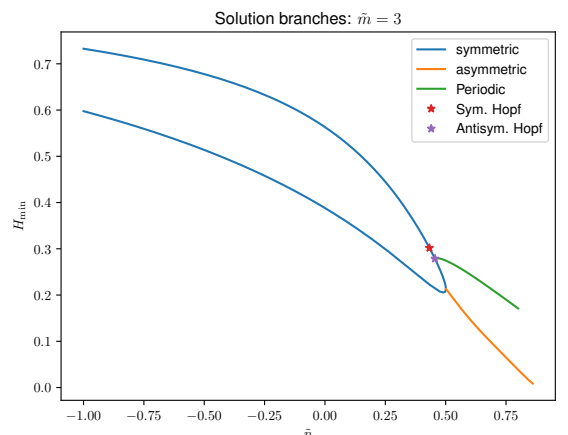

(a)

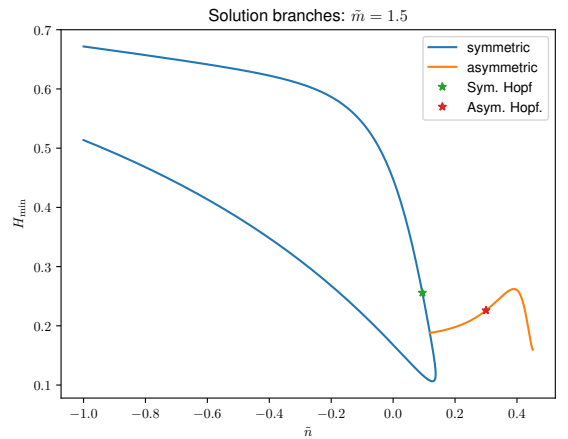

(c)

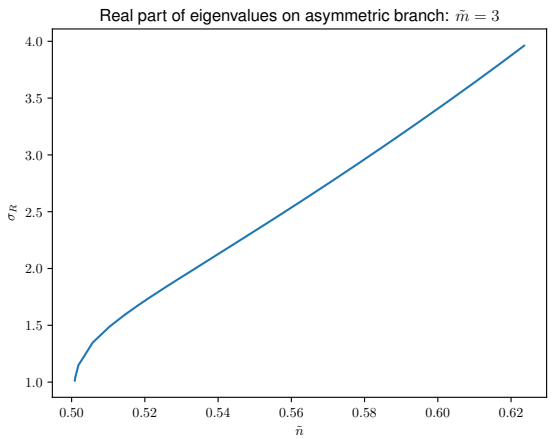

(b)

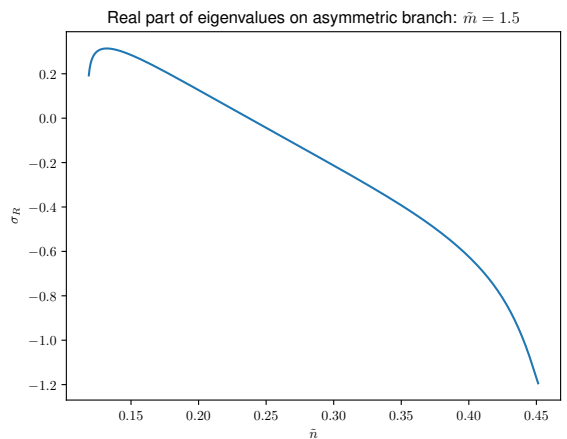

(d)

FiguRE 11. Solution branches for $n=3$ and 1.5, represented by the minimum value $H_{\min }$ of the similarity profile, as function of $\bar{n}$ (left). On the right, the corresponding real part of the largest nontrivial eigenvalue $\nu_{R}$ against $\bar{n}$. For $n=1.5$, a Hopf bifurcation is observed on the asymmetric branch (red star) need new symbol, leading to stable, asymmetric similarity solutions for values of $\bar{n}$ greater than this point. use different symbols for $\mathrm{b} / \mathrm{w}$ ?

encounters the 'symmetric' Hopf bifurcation (blue star), with eigenfunction satisfying $w(-\xi)=\overline{w(\xi)}$, and then an 'antisymmetric' Hopf bifurcation (red plus) with eigenfunction satisfying $w(-\xi)=-\overline{w(\xi)}$. As a function of $\bar{n}$, the symmetric and antisymmetric Hopf bifurcation lines are shown in Fig. 12 in blue and red, respectively.

The ground state solution $H_{0}$ is stable until the largest non-trivial eigenvalue becomes positive, at the symmetric Hopf bifurcation. We use the numerical computation of the trivial eigenvalues $\nu_{T}, \nu_{X}$ as a check on the accuracy of the numerics, which becomes increasingly sensitive for the highly asymmetric solutions. All solution branches here are terminated when $\nu_{T}$ is more than 0.01 different to the expected value of unity.

The fold bifurcation, at wich the $H_{0}$ and $H_{1}$ connect, is marked by a circle. The pitchfork bifurcation, where the asymmetric branch originates (found using the symmetry breaking term), is marked by a cross. We also compute the complex eigenvalues of the asymmetric branch, the largest real part $\nu_{R}$ of which is shown on the right of Fig. 11 for $n=3$ and $n=1.5$. For $n=3, \nu_{R}$ always remains positive, making the corresponding asymmetric branch unstable. For $n=1.5$, on the other hand, $\nu_{R}$ passes through zero at $\bar{n}=0.3 \mathrm{check}$ (red square), where another Hopf bifurcation occurs, which restabilises the 


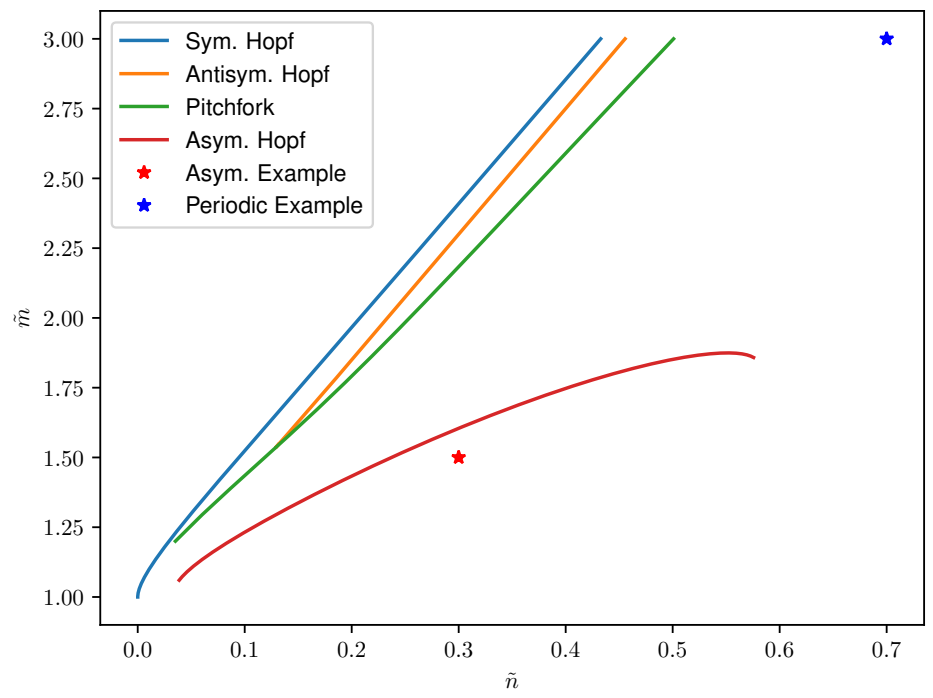

FiguRE 12. Bifurcations in the $(n, \bar{n})$ plane make labels bigger, remove symbols, add fold, look at color scheme

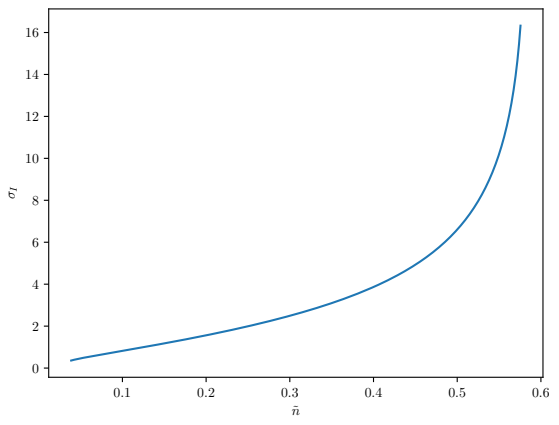

FiguRE 13. The imaginary part of the eigenvalue at the Hopf bifurcation on the asymmetric branch.

asymmetric branch as $\bar{n}$ increases. By numerical continuation we trace out the location of this new Hopf bifurcation in the $n, \bar{n}$ plane, thus finding a region in which stable asymmetric similarity solutions exist (orange line in Fig. 12).

This curve has a turning point at $\tilde{m} \lesssim 1.87$, indicating a maximum value of $n$ for which stable asymmetric similarity solutions exist. As $\bar{n}$ increases further, the imaginary part of the eigenvalue grows large, as seen in Fig. 13. It is not clear if there is another bifurcation at this point, or if the limits of the numerical scheme prevent the curve from being computed further.

\section{A.3. Computation of periodic orbits}

Periodic branches may be computed by using the second, nonlinear method, that is, explicitly coding the finite-difference discretised version of the PDE. In this case 
we restrict ourselves to $\tilde{m}=3$ and focus on the branch of solutions leading off the antisymmetric Hopf bifurcation, as seen in Fig. 11(a).

Using 1241 points and a domain size of $L=20$, the two Hopf bifurcations on the ground state solution branch are found to good accuracy. Then continuing off the antisymmetric Hopf bifurcation, we find a branch of periodic solutions for decreasing $\bar{n}$. A representative solution is plotted for $n=3, \bar{n}=0.7$ in Fig. 2(b). In each period, the value of $H_{\min }(\tau)$ has two minima and two maxima (see inset), as the solution alternates between creating a large undulation to the left and to the right.

\section{REFERENCES}

Angenent, S. B., Aronson, D. G., Betelu, S.I. \& Lowengrub, J. S. 2001 Focusing of an elongated hole in porous medium flow. Physica D 151, 228.

Arnold, V. I. 1984 Catastrophe Theory. Springer.

Aronson, D. G. 2016 The focusing problem for the porous medium equation: Experiment, simulation and analysis. Nonlin. Anal. 137, 135.

Becker, J., Grün, G., Seemann, R., Mantz, H., Jacobs, K., Mecke, K. R. \& Blossey, R. 2003 Complex dewetting scenarios captured by thin-film models. Nature Mat. 2, 59.

Bernoff, A. J., Bertozzi, A. L. \& Witelski, T. P. 1998 Axisymmetric surface diffusion: Dynamics and stability of self-similar pinch-off. J. Stat. Phys. 93, 725-776.

Bertozzi, A. L. \& Pugh, M. C. 1994 The lubrication approximation for thin viscous films: the moving contact line with a 'porous media' cut off of van der waals interactions. Nonlinearity 7, 1535.

Blossey, R. 2012 Thin liquid films. Springer.

Bonn, D., Eggers, J., Indekeu, J., Meunier, J. \& Rolley, E. 2009 Wetting and spreading. Rev. Mod. Phys. 81, 739-805.

Brenner, M. P., Lister, J. R. \& Stone, H. A. 1996 Pinching threads, singularities and the number $0.0304 \ldots$ Phys. Fluids 8, 2827.

Chen, Y.-J. \& Steen, P. H. 1997 Dynamics of inviscid capillary breakup: collapse and pinchoff of a film bridge. J. Fluid Mech. 341, 245-267.

Снортиiк, M. W. 1993 Universality and scaling in gravitational collapse of a massless scalar field. Phys. Rev. Lett. 70, 9.

Cohen, I., Brenner, M. P., Eggers, J. \& Nagel, S. R. 1999 Two fluid drop snap-off problem: experiment and theory. Phys. Rev. Lett. 83, 1147-1150.

Craster, R. V. \& Matar, O. K. 2009 Dynamics and stability of thin liquid films. Rev. Mod. Phys. 81, 1131-1198.

Dallaston, M. C., Fontelos, M. A., Tseluiko, D. \& Kalliadasis, S. 2018 Discrete selfsimilarity in interfacial hydrodynamics and the formation of iterated structures. Phys. Rev. Lett. 120, 034505.

Dallaston, M. C., Tseluiko, D., Zheng, Z., Fontelos, M. A. \& Kalliadasis, S. 2017 Self-similar finite- time singularity formation in degenerate parabolic equations arising in thin-film flows. Nonlinearity 30, 2647.

Day, R. F., Hinch, E. J. \& Lister, J. R. 1998 Self-similar capillary pinchoff of an inviscid fluid. Phys. Rev. Lett. 80, 704-707.

Drazin, P. G. 1992 Nonlinear systems. Cambridge University Press, Cambridge.

EgGers, J. 1993 Universal pinching of 3D axisymmetric free-surface flow. Phys. Rev. Lett. 71, 3458-3460.

EgGers, J. 2012 Stability of a viscous pinching thread. Phys. Fluids 24, 072103.

EgGers, J. 2018 The role of singularities in hydrodynamics. Phys. Rev. Fluids 3, 110503.

EgGers, J. 2020 Self-similar structure of optical caustics.

Eggers, J. \& Fontelos, M. A. 2009 The role of self-similarity in singularities of partial differential equations. Nonlinearity 22, R1-R44.

Eggers, J. \& Fontelos, M. A. 2015 Singularities: Formation, Structure, and Propagation. Cambridge University Press, Cambridge. 
Eggers, J., Grava, T., Herrada, M. A. \& Pitton, G. 2017 Spatial structure of shock formation. J. Fluid Mech. 820, 208-231.

Eggers, J., Herrada, M. A. \& SnoeiJer, J. H. 2020 Self-similar breakup of polymeric threads as described by the oldroyd-b model. J. Fluid Mech. 887, A19-1.

Eggers, J., Hoppe, J., Hynek, M. \& Suramlishvili, N. 2015 Singularities of relativistic membranes. Geometric Flows 1, 17-33.

Fontelos, M. A. \& WAng, Q. 2020 Discrete selfsimilarity in the formation of satellites for viscous cavity break-up. preprint .

Frisch, U. 1995 Turbulence. Cambridge University Press, Cambridge.

Giga, Y. \& Kohn, R. V. 1985 Asymptotically self-similar blow-up of seminlinear heatequations. Comm. Pure Appl. Math. 38, 297.

Grauer, R. \& Sideris, T. C. 1991 Numerical computation of 3d incompressible ideal fluids with swirl. Phys. Rev. Lett. 67, 3511.

Grava, T., Eggers, J. \& Klein, C. 2016 Shock formation in the dispersionless kadomtsevpetviashvili equation. Nonlinearity 29, 1384.

Herrada, M.A. \& Montanero, J.M. 2016 A numerical method to study the dynamics of capillary fluid systems. J. Comp. Phys. 306, 137-147.

Nore, C., Abid, M. \& Brachet, M.-E. 1997 Kolmogorov turbulence in low-temperature superflows. Physical Review Letters 78, 3896.

Nye, J. 1999 Natural Focusing and Fine Structure of Light: Caustics and Wave Dislocations. Institute of Physics Publishing, Bristol.

Pomeau, Y., Le Berre, M., Guyenne, P. \& Grilli, S. 2008 Wave-breaking and generic singularities of nonlinear hyperbolic equations. Nonlinearity 21, T61-T79.

Popinet, S. 2015 Basilisk c libraries. Last accessed on 2020-07-20.

Pumir, A., Shraiman, B. I. \& Siggia, E. D. 1992 Vortex morphology and Kelvin's theorem. Phys. Rev. A 45, R5351.

Witelski, T. P. \& Bernoff, A. J. 1999 Stability of self-similar solutions for van der waals driven thin film rupture. Phys. Fluids 11, 2443.

Witelski, T. P. \& Bernoff, A. J. 2000 Dynamics of three-dimensional thin film rupture. Physica D 147, 155-176.

Zhang, W. W. \& Lister, J. R. 1999 a Similarity solutions for capillary pinch-off in fluids of differing viscosity. Phys. Rev. Lett. 83, 1151-1154.

Zhang, W. W. \& Lister, J. R. $1999 b$ Similarity solutions for van der waals rupture of thin film on a solid substrate. Phys. Fluids 11, 2454-2462. 